山्山 FRANÇAISE

$>\mathrm{DE}$

필 PÉDAGOGIE

\section{Revue française de pédagogie}

Recherches en éducation

171 | avril-juin 2010

La mixité scolaire : une thématique (encore)

d'actualité ?

Demailly Lise. Politiques de la relation. Approche sociologique des métiers et activités professionnelles relationnelles

Villeneuve d'Ascq : Presses universitaires du Septentrion, 2008, 373 p.

\title{
Annette Gonnin-Bolo
}

\section{(2) OpenEdition \\ Journals}

Édition électronique

URL : http://journals.openedition.org/rfp/1962

DOI : 10.4000/rfp.1962

ISSN : 2105-2913

Éditeur

ENS Éditions

Édition imprimée

Date de publication : 1 juillet 2010

Pagination : 150-151

ISBN : 978-2-7342-1187-7

ISSN : 0556-7807

\section{Référence électronique}

Annette Gonnin-Bolo, «Demallıy Lise. Politiques de la relation. Approche sociologique des métiers

et activités professionnelles relationnelles », Revue française de pédagogie [En ligne], 171 | avril-juin 2010, mis en ligne le 12 octobre 2010, consulté le 21 septembre 2020. URL : http://journals.openedition.org/ rfp/1962 ; DOI : https://doi.org/10.4000/rfp.1962

Ce document a été généré automatiquement le 21 septembre 2020.

(c) tous droits réservés 


\section{DEMAILly Lise. Politiques de la relation. Approche sociologique des métiers et activités professionnelles relationnelles}

Villeneuve d'Ascq : Presses universitaires du Septentrion, 2008, 373 p.

Annette Gonnin-Bolo

\section{RÉFÉRENCE}

Demailly Lise. Politiques de la relation. Approche sociologique des métiers et activités professionnelles relationnelles. Villeneuve d'Ascq : Presses universitaires du Septentrion, $2008,373 \mathrm{p}$.

1 Partant du constat du développement quantitatif des «métiers de la relation » et de la place de plus en plus importante de la «relation psychologique » au sein des rapports sociaux et de la sociologie du travail, Lise Demailly propose une synthèse sur les interrogations, les «énigmes» que cela pose pour l'organisation sociale, pour les pratiques professionnelles et pour l'interprétation sociologique. S'appuyant sur un ensemble de travaux qu'elle a menés dans différents secteurs professionnels (formation, enseignement, travail social, santé et santé mentale, mais aussi services et commerce) et sur sa connaissance approfondie de ces secteurs, elle nous offre un ensemble de réflexions qui dépassent le champ précis de l'ouvrage.

2 Posant d'entrée la question du champ couvert par l'ouvrage, elle définit des modélisations possibles des métiers concernés en croisant deux dimensions : la place relative de la relation par rapport à d'autres outils de travail et la visée de l'action, objet de l'intervention (transformation globale ou transformation partielle d'autrui). L'ouvrage se centre certes sur la spécificité de ces métiers mais s'attache aussi à montrer leur appartenance à des problématiques générales de la sociologie des 
professions et du travail. L'approche de différents secteurs professionnels parait particulièrement fructueuse car l'étude des convergences et différences élargit la réflexion sur le système éducatif souvent pris comme seul objet dans de nombreux travaux.

Dans une première partie, Lise Demailly explore à la fois les dimensions importantes pour comprendre ce que sont ces métiers de la relation aujourd'hui et les cadres théoriques pertinents pour éclairer ce champ professionnel, ou plus exactement les champs professionnels propres à chaque métier évoqué. Cette analyse dense met en évidence la complexité des champs et la difficulté de généralisation des traits retenus. Puis après avoir travaillé la question de la formalisation du travail de relation (compétences, dispositifs, doctrines, évaluation...) elle va au-delà en montrant le mouvement d'enchantement, puis de désenchantement et enfin de "réenchantement " dont ces professions font l'objet, marquant ainsi l'importance du sens accordé au travail dans toute approche des professions. Elle montre la pluralité des modèles dans «les modes de professionnalisation et leurs conditions sociales» (p. 77); la diversité mise en avant permet de construire une méthodologie empirique du travail sociologique sur les groupes professionnels, ce qu'elle nous propose en conclusion (p. 107).

4 L'analyse des pratiques du travail relationnel lui permet de définir une typologie des registres d'action (qui sont au nombre de douze), considérés comme catégorie analytique mais aussi dans leur dynamique historique. Si elle interroge les cadres sociaux, les contextes institutionnels et organisationnels dans lesquels se construisent les pratiques, elle aborde également les pratiques d'un point de vue ethnographique et déplie tout ce qui se joue dans le rapport à autrui : transfert, séduction, suggestion, différents types de consultations cliniques, violences, ambivalences, stigmates, différentes censures (rapport à la transparence), secrets...

5 Quant aux savoirs professionnels et à leur construction sociale (p. 169), elle se place d'un triple point de vue: celui des caractéristiques personnelles des individus (propriétés cognitives, mais aussi affectives, caractérielles et idéologiques), celui des situations professionnelles en interne et de leurs exigences (en particulier du statut des destinataires de la relation) et celui de la reconnaissance sociale et de ses conditions. Elle accorde une place importante et particulièrement intéressante à ce que veut dire «l'expérience » et au rapport théorie/expérience.

6 La deuxième partie de l'ouvrage est consacrée aux mutations que subissent ces métiers, mutations certes communes à beaucoup de professions mais aussi spécifiques aux métiers de la relation dans leur diversité (santé et éducation sont étudiées plus particulièrement). Les nouveaux modes de management, les modes de pilotage (qui jouent sur « l'enrôlement des subjectivités » mais aussi sur l'obligation de résultats), la territorialisation et la contractualisation dans le management public entraînent des jeux d'acteurs différents, l'apparition de segments professionnels nouveaux et mettent en tension une rationalisation qui se veut de plus en plus grande et une prise en compte des diversités individuelles. "Amour du métier et malaise" semblent des caractéristiques dominantes chez les professionnels de ces métiers.

Quant aux liens qui se tissent entre différents secteurs professionnels, Lise Demailly souligne à la fois l'obligation de coordination et les difficultés de mise en œuvre dans l'ensemble des secteurs. Riche d'exemples précis, ce chapitre montre la vitalité des secteurs professionnels et des acteurs concernés et pose le problème des modalités 
susceptibles d'encourager la création de réseaux, de partenariats, sans les scléroser par trop de cadrages institutionnels. N'excluant aucune dimension l'auteur termine par un chapitre sur le rôle d'Internet dans les pratiques professionnelles, mais aussi dans le statut des professionnels et des destinataires. Cet ouvrage extrêmement riche présente quatre niveaux d'intérêt :

- une connaissance approfondie et multidimensionnelle de ce que sont aujourd'hui les métiers et activités professionnelles de la relation;

- des apports théoriques car les analyses sont l'occasion de faire le point sur certaines notions parfois utilisées trop rapidement comme la «psychologisation » de la société, la désinstitutionalisation ou la place du genre dans les évolutions des professions. On retrouve là la rigueur de pensée de l'auteur qui n'emboîte pas le pas à certains « prêts à penser » contemporains;

- des méthodologies et des constructions typologiques qui peuvent être utiles à tout chercheur étudiant des champs professionnels ;

- enfin Lise Demailly nous donne à voir ce qu'est le travail du sociologue, sur le terrain. Le passage de l'entretien avec le « Dadarabe » de Madagascar est un exemple particulièrement savoureux d'une démarche ethnologique.

8 Alliance d'un travail de réflexions théoriques rigoureuses, mais jamais enfermées dans des cadres rigides, de résultats et d'exemples de recherches, voire de situations vécues, qui donnent chair à l'ouvrage, cet ensemble très complexe et très fouillé mérite d'être lu en plusieurs temps: un temps pour comprendre la complexité de cette tentative de synthétiser ce que peuvent être les métiers et activités professionnelles et relationnelles et ensuite par passages ciblés selon le champ de préoccupation du lecteur et ses interrogations du moment.

\section{AUTEURS}

\section{ANNETTE GONNIN-BOLO}

Université de Nantes 